\title{
Significance bias: an empirical evaluation of the oral health literature
}

\author{
Edwin Kagereki ${ }^{*}$, Joseph Gakonyo ${ }^{2}$ and Hazel Simila ${ }^{2}$
}

\begin{abstract}
Background: The tendency to selectively report "significant" statistical results (file-drawers effect) or run selective analyses to achieve "significant" results (data-dredging) has been observed in many scientific fields. Subsequently, statistically significant findings may be due to selective reporting rather than a true effect. The p-curve, a distribution of $p$-values from a set of studies, is used to study aspects of statistical evidence in a scientific field. The aim of this study was to assess publication bias and evidential value in oral health research.

Methods: This was a descriptive and exploratory study that analysed the $p$-values published in oral health literature. The National Library of Medicine catalogue was searched for journals published in English, indexed in PubMed and tagged with dentistry Medical Subject Headings (MeSH) words. Web scraping for abstracts published between 2004 and 2014 was done and all $p$-values extracted. A p-curve was generated from the $p$-values and used for analysis. Bayesian binomial analysis was used to test the proportion of the $p$-values on either side of the 0.05 threshold (test for publication bias) or the 0.025 threshold (test for evidential value). The tacit assumption was that significant $p$-values reported were the result of publication bias.

Results: The present study found the use of $p$-values in a total of 44,315 $p$-values published in 12,440 abstracts. Two percent of the $p$-values were inaccurately reported as zero or $\geq 1$. The $\mathrm{p}$-curve was right skewed, with an intriguing bi-modality. The distribution of the $p$-values is also unequal on either side of 0.025 and 0.045 of the p-curve.
\end{abstract}

Conclusions: This study found evidence of data-dredging, publication bias and errors in the dental literature. Although the present study was conducted on abstracts, the findings highlight a subject that should be researched in future studies that would consider the various factors that may influence $p$-values.

Keywords: $P$-value, File drawer effect, Data-dredging, Evidential value, Significance bias

\section{Background}

Goodhart's law states that "When a measure becomes a target, it ceases to be a good measure" [1]. Prevailing evidence in scientific publications corroborates this law, with many journals selectively publishing statistically significant results [2,3]. Publication bias is a phenomenon that arises when statistical significance strongly influences the chances of publication. With the ever-increasing pressure to publish or perish, researchers start considering bending the rules to increase the chances of their work getting published [4].

\footnotetext{
*Correspondence: kabethte@gmail.com

'Department of Periodontology/Community and Preventive Dentistry,

University of Nairobi, P.O. BOX 30197-00100, Nairobi, Kenya

Full list of author information is available at the end of the article
}

A notable negative effect of publication bias is the influence it has on meta-analysis [5]. The latter combines the quantitative evidence from related studies to summarize a whole body of research on a particular question which is the guiding principle in evidence based medicine. It therefore follows that if the published research findings are biased, then the conclusions drawn might be flawed. A recent study done in Yale claimed to show evidence of an association between dental $\mathrm{x}$-rays and intracranial meningioma [6]. However, upon further interrogation of the study, irreconcilable data problems highlighted serious flaws in the study that render the conclusions invalid [7]. Publication bias also affects the effectiveness of replication as a tool of validation of scientific findings [8]. This bias has been widely studied in the context of null hypothesis 
significance testing (NHST) whereby the pre-dominant measure of the scientific decisions is the $p$-value. The role of NHST has been questioned on epistemological reasons, with some authors suggesting the abandonment of $p$-values $[9,10]$. Some journals like Epidemiology [11] and Basic and applied psychology [12] have taken a principled stand against them.

The NHST was introduced by R. A. Fisher, Jerzy Neyman and Egon Pearson and has since been widely adopted as the "gold standard" in hypothesis testing [13]. The probability of getting an outcome from the null hypothesis that is as extreme as (or more extreme than) the actual outcome, is called the $p$-value. If the $p$-value is very small, conventionally less than $5 \%$, then the null hypothesis is rejected. This arbitrary cut-off has led to the scientifically dubious practice of regarding "significant" findings as more valuable, reliable, and reproducible [14]. In reality, there can be many possible $p$-values for any set of data; depending on how and why the data was generated [15]. Furthermore, $p$-values also depend on the tests that the analyst decides to use, making them highly subjective [16]. Thus $p$-values present fundamental logical problems which are highlighted below to induce the readers' curiosity.

To begin with, the significance tests are often misunderstood and misinterpreted [17]. For example, it is often equated with the strength of a relationship, but a tiny effect size can have very low $p$-values with a large enough sample size. Similarly, a low $p$-value does not mean that a finding is of major clinical or biological significance [18]. Subsequently a $p$-value alone does not reveal relevant information concerning effect sizes and or even the direction of the effect. It is therefore advisable that $p$-values are interpreted in context.

In addition, the analyst has an option to apply alternative methods and tests to get intended results (usually statistically significant findings) without a prior analysis plan to answer the scientific question at hand [16]. In this way the analyst is able to control the false alarms on the basis of his/her intention, not on the basis of the research problem. This debate may continue for a long time, as it touches on philosophy of science.

Researchers have studied various methods in which publication bias has been perpetrated. One such method is data-dredging (also termed as snooping, fishing, significance-chasing or double-dipping) [19]. This entails multiple attempts at data analysis to achieve desired results. For example, an analyst may use partial data to decide whether to or not to continue with the analysis. It may also involve manipulation of variables post-analysis to achieve desirable and pre-determined results [16]. For instance dropping outliers, splitting or regrouping treatment groups or variable transformation. Another way in which publication bias may arise is the 'file-drawer effect'. This is a phenomenon in which researchers tend to forward studies with significant results for publication, while withholding those with non-significant findings [19].

A p-curve is the distribution of $p$-values for a set of studies which assumes that the distribution of $p$-values is a random variable with some level of uncertainty [20]. This set of $p$-values can form a probability distribution with all possible outcomes and their corresponding probabilities. Thus in reality, the candidate $p$-values form a finite continuum from zero to one, both zero and one being excluded. This curve has been adopted as a tool in the study of evidence in various scientific fields $[19,21]$.

One application of the p-curve is to detect presence of publication bias. A sharp drop of the p-curve for values above the significance level illustrates this bias [18]. This curve may also be used to detect data-dredging. Here, the assumption is that if researchers turn a nonsignificant $p$-value into a significant one, then the shape of this curve will be altered around the perceived significance threshold [14, 17].

Moreover, the p-curve has been used to study evidential value in a set of studies [14, 17]. This is considered to be present when the published evidence for a specific hypothesis consistently suggests that the effect truly exists across a set of studies. When the true effect is strong, researchers are more likely to obtain very low $p$-values $(p<0.001)$ than moderately low $p$-values $(p<0.01)$, and less likely to obtain non-significant $p$-values $(p>0.05)$ [18]. Therefore, as the true effect size increases the p-curve becomes more skewed to the right [19]. Binomial tests have previously been used to assess existence of evidential value and data-dredging $[14,17]$. To achieve this goal, the significant $p$-values are binned into two groups; $0<p \leq 0.025$ (lower bin) and $0.026 \leq p \leq 0.05$ (upper bin). The assumption here is that if evidential value is present, the expected number of $p$-values in the lower bin should be equal to or greater than that in the upper bin. Conversely, if there are more $p$-values in the upper bin, then data-dredging is a plausible explanation [21].

It has however been noted that the method proposed above only detects severe data-dredging but may fail to detect modest levels [18]. A more sensitive approach would be to narrow down on the $p$-values close to 0.05 where it is expected that the signals of data-dredging would be strongest. It has been established that p-hackers have limited ambition and tend to alter only the $p$-values close to the 0.05 threshold [15]. To do this the $p$-values close to 0.05 are divided into two bins, one between 0.04 and 0.045 (lower bin), and the upper bin to contain $p$ values between 0.046 and 0.05 . Ideally the two bins should be equal if there is no manipulation of the $p$ values. Comparing the proportion of the $p$-values in the upper bin to those in the lower bin is a more sensitive test of data-dredging [17]. 
A subtle technique observed in data-dredging is strategic rounding-off $[18,22]$. In this, $p$-values with two to three decimal places above the threshold are conveniently rounded-down to achieve the statistically significant threshold. For instance, if the obtained value is below 0.054 then it is rounded-down to 0.05 . To test the presence of this strategic rounding-off, the proportion of marginally significant $p$-values ( $p$-values between 0.045 and 0.049) are compared with the marginally nonsignificant $p$-values ( $p$-values between 0.051 and 0.054 ). It therefore follows that if the marginally non-significant $p$-values are fewer than the marginally significant $p$ values, then there is evidence of strategic rounding off.

The p-curve therefore is a useful tool to help researchers in a field to assess possible ways in which $p$ values could be dragging scientific processes down by biased reporting of the results [23]. The aim of this study was to assess file-drawer effect, data-dredging, strategic rounding-off and evidential value in oral health literature by studying the p-curve. The tacit assumption here was that these factors affect the reported $p$-values. It is hoped that the findings will contribute to the debate on the alternative methods to the NHST.

\section{Methods}

A descriptive and exploratory study analysed the $p$ values published in oral health literature from January 2004 through December 2014. Web scraping for the abstracts published in all the volumes was done and all the $p$-values extracted. A total of 31 journals out of an initial 789 entries were used for the analysis.

\section{Search strategy}

The National Library of Medicine (NLM) catalogue was searched for journals published in English, indexed in PubMed and tagged with dentistry MeSH (Medical Subject Headings) words (MeSH Unique ID: D003813). This search was done with the NLM Catalog Advanced Search Builder using the MeSH word for the entries on "MeSH Major topic" OR "MeSH Terms" OR "MeSH Subheading". Filters activated were: "Only PubMed journals" and "English".

The results of the search were collected in a collection file and downloaded as a comma separated value (.csv) file. A total of 789 entries were identified. All the duplicated entries and journals missing with any missing volume within the study period were excluded. This is summarized in Fig. 1. All the researchers were involved in the search any arising disputes was resolved through a consensus by all of them.

\section{Journals included}

The following journals were included in this study:

J Contemp Dent Pract,Br J Oral Maxillofac Surg,Int J
Oral Maxillofac Surg,J Clin Dent,Int J Dent Hyg,BMC
Oral Health,Oral Health Prev Dent,Community Dent
Oral Epidemiol,J Oral Sci,Braz Oral Res,J Adhes Dent,J
Clin Pediatr Dent,J Craniofac Surg,Am J
Dent,Community Dent Health,Gerodontology,J Oral
Maxillofac Surg,Int Endod J,Eur J Orthod,J Oral
Implantol,Gen Dent,J Endod,J Clin Periodontol,J Dent,J
Periodontol,Caries Res,J Periodontal Res,Arch Oral Biol,J

J Contemp Dent Pract,Br J Oral Maxillofac Surg,Int J Oral Maxillofac Sung, Clin Dent, Int J Dent Hyg,BMC Oral Epidemiol,J Oral Sci,Braz Oral Res,J Adhes Dent,J Clin Pediatr Dent,J Craniofac Surg,Am J

Dent,Community Dent Health, Gerodontology,J Oral Implantol,Gen Dent,J Endod,J Clin Periodontol,J Dent,J Periodontol,Caries Res,J Periodontal Res,Arch Oral Biol,J

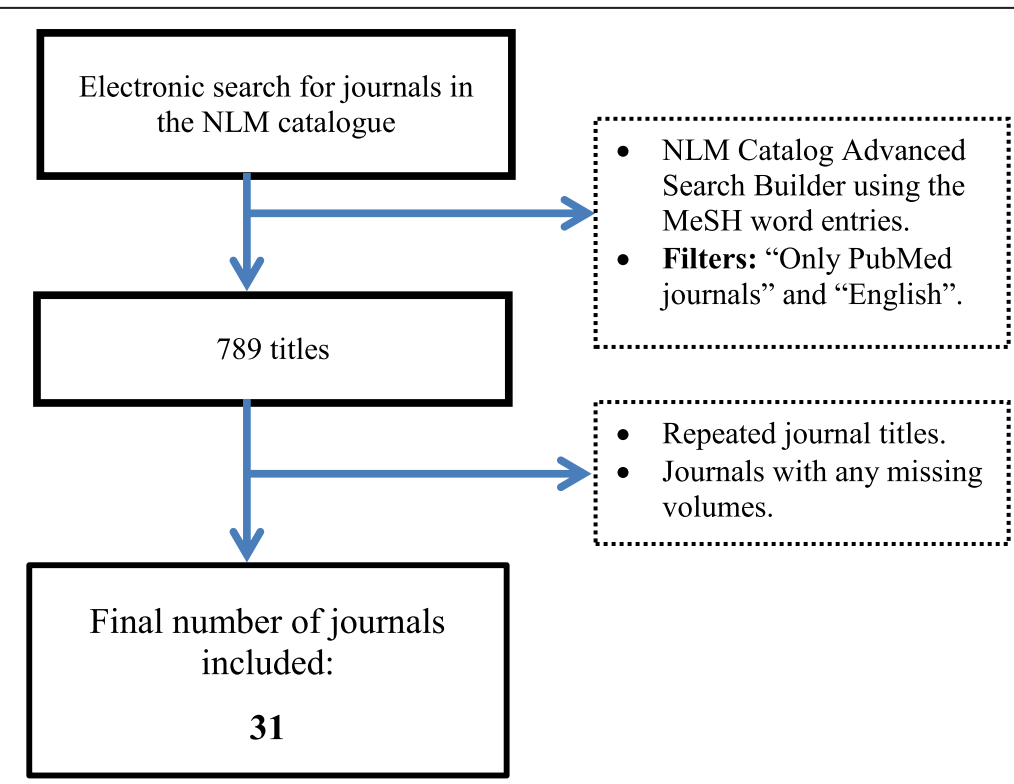

Fig. 1 Search strategy. The National Library of Medicine (NLM) catalogue was searched for journals published in English, indexed in PubMed and tagged with dentistry MeSH (Medical Subject Headings) words (MeSH Unique ID: D003813). Repeated entries and journals with missing volumes within the study period were excluded 
Prosthet Dent,Int Dent J,Br Dent J,Angle Orthod, Clin Implant Dent Relat Res,Int J Dent Hyg and Oral Health Prev Dent.

\section{Statistical analysis}

The following variables were collected: title of the journal, PubMed ID, year of publication, $p$-values, title of article and the abstract using $R$ package (Version 3.1.2, $\mathrm{R}$ Development Core Team, Vienna, Austria). All the data analysis was done using $\mathrm{R}$ and the relevant packages. The $\mathrm{R}$ code used is provided as Additional file 1 .

To test for the distribution of the $p$-values across the thresholds, Bayesian binomial test was used to estimate the $95 \%$ high definition intervals (HDI) estimated. A non-informative prior was used based on the distribution of Beta $(1,1)$ distribution.

The study examined all the $p$-values that reported in abstract of all the selected journal volumes between 2004 and 2014. However, the $p$-values erroneously reported as zero or one and were excluded from the pcurve analysis. A summary of the research process is depicted in Fig. 1.

\section{Results}

\section{Number of reported $p$-values}

In this study, a total of 44,315 $p$-values were abstracted from 12,440 abstracts. The paper with the maximum number of $p$-values had 48 , with most of the other papers reporting a single $p$-value. There were $157(<1 \%) p$ values reported as one and 617(1\%) $p$-values reported as zero. Further, $10,960(25 \%) p$-values were reported as exactly 0.05 . The distribution of the reported $p$-values is summarized in Fig. 2.

\section{Assessment of the p-curve for selection bias/file-drawer effect}

There was an over-abundance of $p$-values below the 0.05 threshold as illustrated in Fig. 2a. A bi-modality was observed in the distribution of all $p$-values: around 0 and also around the significance threshold of .05 as shown in Fig. $2 \mathrm{~b}$.

\section{Assessment of the p-curve for data-dredging and evidential value}

To test for evidential value the proportion of the $p$-values below the 0.05 threshold were divided into two bins. There were 22,468 $p$-values in the lower bin $(0-0.025)$, while 15,414 $p$-values were in the upper bin $(0.026-0.05)$. Bayesian binomial test was used to test equality of these proportions. The estimated percentage of the lower $p$-values (up to 0.025 ) was $59.3 \%[58.8,59.5]$. The relative frequency of the lower $p$-values was more than 0.5 estimated by a probability of $>0.999$ and less than 0.5 by a probability of $<0.001$.

To test for data-dredging, the $p$-values close to the 0.05 threshold were divided into two bins. There were $1224 p$-values in the lower bin (0.04-0.45) and $15,414 \quad p$-values in the upper group (0.046-0.05). Bayesian binomial test was used to test the equality of these proportions. This resulted in an estimated proportion of 0.097 [0.092, 0.102] for the lower bin. The relative frequency of the lower bin was more than 0.5 by a probability of $<0.001$ and less than 0.5 by a probability of $>0.999$.

\section{Strategic rounding of $p$-values to show significance in reported results}

A comparison between the proportion of the marginally significant $p$-values ( $p$-values between 0.040 and 0.049 )
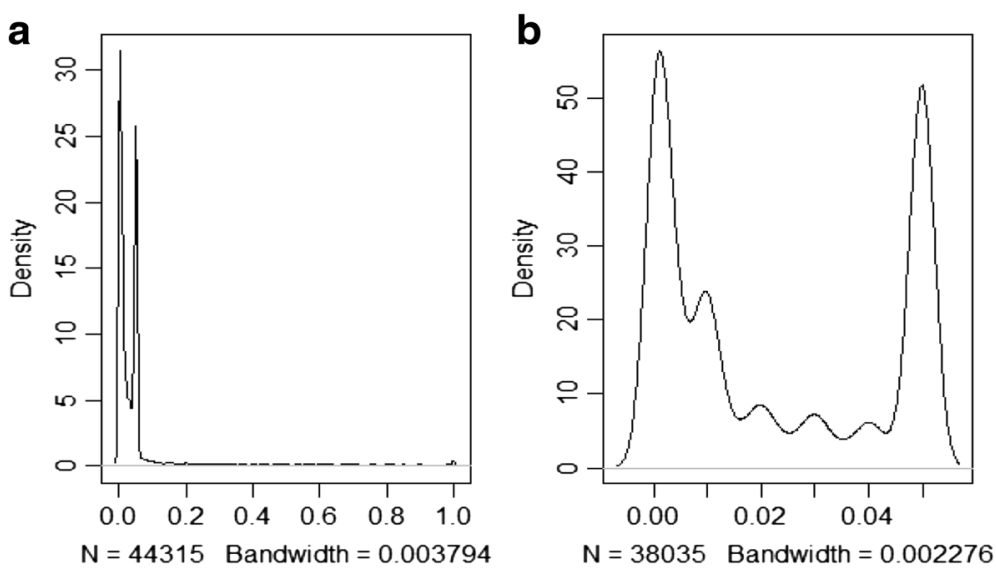

Fig. 2 The p-curve of the 44,315 p-values studied. The curve on the left a illustrates the overabundance of the $p$-values below the 0.05 threshold. The curve on the right $\mathbf{b}$ is a closer look at the $p$-values below the 0.05 threshold illustrating a bi-modal distribution of the $p$-values; one peak close to zero and the other close to the conventional significant threshold of 0.05 
and the proportion of the marginally non-significant $p$ values ( $p$-values between 0.051 and 0.054 ) was done. The marginally significant $p$-values were $15,334(99.19 \%)$ as compared to the marginally insignificant $p$-values 125 $(0.81 \%)$. Bayesian binomial to test the difference between these two proportions estimated the proportion of the marginally significant to be $0.992[0.99,0.993]$. The relative frequency of the marginally significant was more than 0.5 by a probability of $>0.999$ and less than 0.5 by a probability of $<0.001$.

\section{Reported p-values across the various disciplines in dentistry}

A total of 13 dental specialties were considered in this study. This was guided by the MeSH for each journal. Consistently, significant $p$-values were reported across the disciplines. Data-dredging was evident in all the disciplines although dental materials had proof of evidential value (Table 1 ).

\section{Discussion}

In studying the p-curve we observed that it was generally right skewed with two peaks: one close to 0 and the other near the significance threshold of 0.05 . One possible explanation of this finding is based on the general assumption that researchers manipulate their findings to increase chances of their work getting published (strategic reaction to publication bias). The high number of small $p$-values (less than 0.05) observed in the present study across the range of the oral health specialties (with the exception of dental materials) could also imply that a majority of researchers predominantly study phenomena where an actual difference is known to already exists (evidential value) [20, 24]. It is therefore necessary to conduct further investigations on the research questions studied in oral health.

Statistical power considerations associated with statistical tests of hypotheses relate to the likelihood of correctly rejecting the tested hypotheses, given a particular beta level, alpha level, effect size and sample size. Consequently, an intimate relationship between these four measures exists. Small $p$-values would therefore result from small study effects, large samples, high power or a combination of them. It is therefore possible that these factors may lead to the right skew of the p-curve. Future research is necessary to investigate the foregoing and to secure more evidence on the prevalence of congruence errors in oral health literature [5].

The authors noted that in some of the journal articles included in the present study reported multiple $p$-values suggesting that multiple hypotheses were tested simultaneously. Testing several independent null hypotheses and maintaining the threshold at 0.05 for each comparison, the chance of obtaining at least one "statistically significant" result is greater than $5 \%$ (even if all null hypotheses are true). Conventionally, where multiple testing is done, additional adjustments are done to alleviate the critical values for the hypotheses of interest, and make rejection of these hypotheses more likely. Therefore without further analysis, it is not possible to disregard the possibility that failure to compensate for multiple comparisons could have resulted in the overabundance in the small $p$-values [25].

The distribution of the $p$-values also, suggests evidence of data-dredging. The higher proportion of the $p$-values close to the 0.05 threshold may suggest that the researchers may have manipulated the $p$-values to get close to the threshold. The results of the present study are in accordance with the majority of the previous findings in

Table 1 Tests for evidential value and data-dredging across dental specialties. Evidence of data-dredging was there across the disciplines

\begin{tabular}{llllllll}
\hline Discipline & Frequency & 0 to 0.025 & $0.026-0.05$ & Test for evidential value & $0.04-0.045$ & $0.046-0.05$ & Test for data-dredging \\
\hline General Dentistry & $10948(25 \%)$ & 5366 & 4108 & $0.57[0.62,0.64]$ & 212 & 3364 & $0.059[0.052,0.067]$ \\
Surgery & $8605(19 \%)$ & 4372 & 2564 & $0.63[0.62,0.64]$ & 348 & 1523 & $0.19[0.17,0.20]$ \\
Public Health Dentistry & $1805(4 \%)$ & 1122 & 478 & $0.70[0.68,0.72]$ & 62 & 315 & $0.17[0.13,0.20]$ \\
Dental Materials & $821(2 \%)$ & 325 & 392 & $0.45[0.42,0.49]^{\ddagger}$ & 1 & 355 & $0.0046[0.00015,0.013]$ \\
Pedodontics & $490(1 \%)$ & 246 & 184 & $0.57[0.53,0.62]$ & 22 & 114 & $0.17[0.11,0.23]$ \\
Gerodonlogy & $922(2 \%)$ & 445 & 316 & $0.58[0.55,0.62]$ & 26 & 223 & $0.11[0.071,0.15]$ \\
Endodontics & $5456(12 \%)$ & 2309 & 2468 & $0.48[0.47,0.50]$ & 109 & 2133 & $0.049[0.040,0.058]$ \\
Orthodontics & $2265(5 \%)$ & 1229 & 736 & $0.63[0.60,0.65]$ & 80 & 545 & $0.13[0.10,0.16]$ \\
Implantology & $553(1 \%)$ & 267 & 170 & $0.61[0.57,0.66]$ & 19 & 113 & $0.15[0.091,0.21]$ \\
Periodontics & $8770(20 \%)$ & 4666 & 3048 & $0.60[0.59,0.62]$ & 298 & 2074 & $0.13[0.11,0.14]$ \\
Cariology & $945(2 \%)$ & 565 & 280 & $0.67[0.64,0.70]$ & 24 & 189 & $0.12[0.075,0.16]$ \\
Oral Hygiene & $438(1 \%)$ & 242 & 146 & $0.62[0.58,0.67]$ & 16 & 89 & $0.16[0.091,0.23]$ \\
Prosthodontics & $2231(5 \%)$ & 1311 & 493 & $0.73[0.71,0.75]$ & 33 & 338 & $0.09[0.062,0.12]$ \\
\hline
\end{tabular}

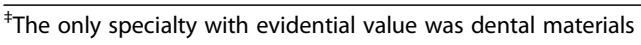


other scientific fields $[3,20]$. The un-equal distribution of the $p$-values between 0.045 and 0.049 as compared to those between 0.051 and 0.055 could probably be due to the rounding down of the values between 0.051 and 0.054 to achieve the significance value of 0.05 [17].

The analysis presented in this study is rather novel and exploratory and may contribute to the discussion whether we should substantially change the way we do statistics. Further they support the suggestion that many research findings maybe false [2]. On a wider scope, these findings raise many questions on the evidence reported in oral health. One such inquiry is whether there is congruence between the power, effect size, $p$-value and test statistic or repetition of the research hypotheses. Further, one may wish to know if there exists bias where other inferential methods have been used.

\section{Conclusion}

This study found presence of evidential value, datadredging, publication bias in the oral health literature. The fact that researchers may wish to publish their significant findings in their abstracts while leaving the nonsignificant results is an inherent limitation of the present study. Additionally, the numerous small $p$-values observed may be attributed to multiple testing. The foregoing can be overcome in future studies by including the full research papers. With the original data, a re-run of all tests would reveal presence of bias where other inferential methods have been used and also identify incongruences in the statistical evidence reported.

\section{Additional file}

Additional file 1: Functions to scrape P-values from Pubmed abstracts. (TXT $5 \mathrm{~kb}$ )

\section{Competing interests}

Authors EK, JG, HS state that there are no conflicts of interest.

\section{Authors' contributions}

All authors contributed to study concept, review and approval of the final manuscript. EK contributed to data acquisition and statistical analysis.

\section{Acknowledgements}

The authors would wish to acknowledge the support accorded to them by the University of Nairobi, School of Dental Science administration during authorship of this paper. We further wish to appreciate the editorial team of $\mathrm{BMC}$ oral health for their objective reviews.

\section{Author details}

'Department of Periodontology/Community and Preventive Dentistry, University of Nairobi, P.O. BOX 30197-00100, Nairobi, Kenya. ${ }^{2}$ Department of Conservative and Prosthetic Dentistry, University of Nairobi, P.O. BOX 30197-00100, Nairobi, Kenya.

Received: 10 August 2015 Accepted: 21 April 2016

Published online: 05 May 2016

\section{References}

1. Alec C et al. Goodhart's Law: Its Origins, Meaning and Implications for Monetary Policy. A Festschrift in honour of Charles Goodhart held on 15-16 November 2001 at the Bank of England.

2. Fanelli D. Negative results are disappearing from most disciplines and countries. Scientometrics. 2012;90:891-904.

3. Rosenthal R. The file drawer problem and tolerance for null results. Psychol Bull. 1979;86:638-41.

4. Sterne JA, Egger M, Smith GD. Systematic reviews in health care: investigating and dealing with publication and other biases in metaanalysis. BMJ. 2001;323:101.

5. Ferguson CJ, Heene M. A vast graveyard of undead theories: publication bias and psychological Science's aversion to the null. Perspect Psychol Sci. 2012;7(6):555-61.

6. Claus EB et al. Dental x-rays and risk of meningioma. Cancer. 2012;118(18): 4530-7.

7. Ernest et al. The American Academy of Oral and Maxillofacial Radiology. AAOMR Response to Recent Study on Dental X-ray Risks. April 2012.

8. Ferguson C, Heene M. A vast graveyard of undead theories publication bias and psychologicl Science's aversion to the null. Perspect Psychol Sci. 2012; 7(6):555-61.

9. Wolf PK. Pressure to publish and fraud in science. Ann Intern Med. 1986; 104(2):254-6.

10. Goodman SN. Of P-values and bayes: A modest proposal. Epidemiology. 2001;12(3):295-297.

11. Rothman K. Writing for epidemiology. Epidemiology. 1998;9:333-7.

12. David T. Michael marks basic. Appl Soc Psych. 2015;37:1-2.

13. Leif D. Nelson. False-Positives, p-Hacking, Statistical Power, and Evidential Value. Summer Institute University of California, Berkeley-Haas School of Business; 2014.

14. Nickerson RS. Null hypothesis significance testing: a review of an old and continuing controversy. Psychol Methods. 2005;5:241-301.

15. Kruschke JK. Null hypothesis significance testing. Doing Bayesian data analysis. 2nd ed. CA, USA: Elsevier; 2011. p. 297-331.

16. Gadbury GL, Allison DB. Inappropriate Fiddling with Statistical Analyses to Obtain a Desirable P-value: Tests to Detect its Presence in Published Literature. PLoS ONE. 2012; 7 (10): e46363. doi:10.1371/journal.pone.0046363.

17. Mariscampo EJ, Lalande DR. A peculiar prevalence of $p$ values just below 05. Q Rev Biol. 2012:65:2271-9.

18. Nakagawa S, Cuthill IC. Effect size, confidence interval and statistical significance: a practical guide for biologists. Biol Rev Camb Philos Soc 2007:82:591-605.

19. Simonsohn U, Nelson LD, Simmons JP. P-curve: a key to the file drawer. J Exp Psychol Gen. 2014;143:534-47.

20. Head ML, Holman L, Lanfear R, Kahn AT, Jennions MD. The extent and consequences of P-hacking in science. PLoS Biol. 2015;13(3):e1002106.

21. de Winter JC, Dodou D. A surge of $p$-values between 0.041 and 0.049 in recent decades (but negative results are increasing rapidly too). PeerJ. 2015;3:e733.

22. Regina N. $P$ values, the 'gold standard' of statistical validity, are not as reliable as many scientists assume. Nature. 2014;506:150-2.

23. Ioannidis JPA. Why most published research findings Are false. PLoS Med 2005;2(8):e124

24. Leggett NC, Thomas NA, Loetscher T, Nicholls MER. The life of p: "just significant" results are on the rise. Q J Exp Psychol. 2013;66:2303-9.

25. Noble WS. How does multiple testing corrections work? Nat Biotechnol. 2009:27:1135-7. 